1959 and 1978 at two boatbuilding plants. The a priori hypothesis: leukaemia and lymphoma excesses would be found

Method Standardised mortality ratios (SMR), standardised rate ratios (SRR), and 95\% confidence intervals (CI) were calculated using Washington State rates and a person-years analysis program, LTAS. NET, controlling for age, calendar period, race, and gender. The SRR analysis compared tertiles of estimated cumulative styrene exposure.

Results Overall, 484 cancer deaths occurred (SMR 1.20, CI 1.10-1.31), with excess mortality for respiratory cancers $(\mathrm{n}=$ 171, SMR 1.33, CI 1.14-1.55) and prostate cancer $(\mathrm{n}=41$, SMR 1.44, CI 1.03-1.96). Among 2063 workers highly exposed to styrene and fibreglass there were excesses of mesothelioma (n $=3$, SMR 5.28, CI 1.09-15.4) and ovarian cancer ( $\mathrm{n}=6$, SMR 2.94, CI 1.08-6.41). The SRR analysis did not find strong associations between tertiles of styrene exposure and cancer mortality.

Conclusions We found no excess leukaemia or lymphoma mortality. Unanticipated excess mesothelioma and ovarian cancer mortality are difficult to interpret and could be due to fibreglass exposure or employment elsewhere, or could be chance findings.

\section{IS GENDER ADEQUATELY STUDIED IN AGRICULTURAL WORKERS' HEALTH RESEARCH?}

Rima R Habib, Kareem Elzein, Safa Hojeij. American University of Beirut, Beirut, Lebanon

\subsection{6/oemed-2014-102362.233}

Objectives Gender-sensitive research strategies address men's and women's health problems by identifying physiological, ergonomic and socio-cultural gender characteristics that shape study outcomes. These strategies have been inadequately accounted for in many occupational health researches on agriculture workers. In reviewing the occupational health literature on agriculture workers, this paper assesses the processes employed to analyse how gender affects work-related health outcomes.

Method Peer-reviewed articles concerned with male and female agricultural workers' health and published between 2000 and 2011 in PubMed were evaluated. Articles that use gender stratification were identified and analysed for their approaches toward sampling, data analysis, task differentiation and use of other exposure indicators.

Results Out of 176 articles, only 26 (15\%) analysed the associations between occupational health exposures and health outcomes using gender stratification. Many studies failed to recruit adequate female participants or have marginalised gender at an early stage of the research. The role of females as homemakers was also inadequately conceptualised. Several others did not collect adequate task or exposure information to identify established risk factors relating to study outcomes.

Conclusions Occupational health research on farm workers struggle to incorporate gender analysis into research design and analytical approaches. The role of gender in shaping health outcomes is evident in occupational health research. Developing methodologies, study designs, and analysis that are gendersensitive will improve the quality of research and help tailor sound interventions and policies. This could be through incentives and support from research funding agencies and through incorporating gender perspectives into academic journal editorial policies.

\section{LIFETIME OCCUPATIONAL EXPOSURE TO DIESEL EXHAUST AND BLADDER CANCER AMONG MEN IN NEW ENGLAND}

${ }^{1}$ Stella Koutros, ${ }^{2}$ Margaret Karagas, ${ }^{1}$ Melissa Friesen, ${ }^{3}$ Anjoeka Pronk, ${ }^{4}$ Patricia Stewart, ${ }^{1}$ Dalsu Baris, ${ }^{5}$ Molly Schwenn, ${ }^{2}$ Richard Waddell, ${ }^{6}$ Alison Johnson, ${ }^{5}$ Castine Clerkin, ${ }^{7}$ Karla Armenti, ${ }^{1}$ Joanne Colt, ${ }^{1}$ Debra Silverman. ${ }^{1}$ Division of Cancer Epidemiology and Genetics, National Cancer Institute, Bethesda, MD, USA; ${ }^{2}$ Department of Community and Family Medicine, Geisel School of Medicine at Dartmouth, Hanover, NH, USA; ${ }^{3} T N O$ Quality and Safety, Zeist, The Netherlands; ${ }^{4}$ Stewart Exposure Assessments, LLC, Arlington, VA, USA; ${ }^{5}$ Maine Cancer Registry, Augusta, ME, USA; ${ }^{6}$ Vermont Cancer Registry, Burlington, VT, USA; ${ }^{7}$ New Hampshire Department of Health and Human Services, Concord, NH, USA

\subsection{6/oemed-2014-102362.234}

Objectives We examined the association between lifetime occupational diesel engine exhaust (DEE) exposure and risk of bladder cancer in 1171 cases and 1418 controls in a populationbased case-control study.

Method Lifetime occupational histories combined with additional exposure-oriented questionnaires were administered to obtain detailed information on DEE. We estimated the probability, frequency and intensity of exposure to respirable elemental carbon (REC) $\left(\mu \mathrm{g} / \mathrm{m}^{3}\right)$, a primary surrogate for DEE. Unconditional logistic regression was used to calculate odds ratios (ORs) and $95 \%$ confidence intervals (CIs), adjusting for smoking and other risk factors.

Results DEE was associated with an increased risk of bladder cancer, with the highest level of cumulative REC $\left(>252.8 \mu \mathrm{g} / \mathrm{m}^{3}\right.$ per year) having a $35 \%$ elevated risk $(95 \% \mathrm{CI}=0.86-2.13)$ compared to those with no exposure. Among nonsmokers, we observed a significant trend in risk with increasing cumulative REC (p-trend $=0.03$ ), with heavily exposed subjects having an $\mathrm{OR}=2.80$ (95\% CI $=1.08-7.22$ ). Time-period analyses by decade of first DEE-exposed job showed a statistically significant increased risk among men first exposed in the 1950s (heavily exposed: $\mathrm{OR}=2.73,95 \% \mathrm{CI}=1.29-5.79$, p-trend $=0.009$ ).

Conclusions The overall risk observed is modest, but similar in magnitude to those observed at comparable levels of exposure in previous studies of bladder and lung cancer. Greater risk for those first exposed in the 1950s may reflect secular trends in risk or a long latency for bladder cancer. Our results provide additional evidence that DEE is related to an increased bladder cancer risk.

\section{HOME CARE WORKER ERGONOMIC HAZARDS}

Felipe Tendick Matesanz, Jane Thomason, Marsha Love, Joseph Zanoni. University of Illinois at Chicago School of Public Health, Chicago, USA

\subsection{6/oemed-2014-102362.235}

Objectives Home care workers (HCW) are at high risk of musculoskeletal disorders related to consumer care and housekeeping tasks and need of ergonomic interventions (Arlinghaus, et al., 2013; Baron and Habes, 2004; Hodsonetal et al., 2010). The goal of this project is to explore HCW experiences and perceptions to document potentially hazardous tasks to inform intervention and policy.

Method In 2012 researchers partnered with a labour union (SEIU) representing HCWs to recruit those with at least 3 years of experience with consumers needing moving assistance. Forty six mostly African Americans participated in four focus groups and a validation session. Participants were asked to review a 
listing of common tasks, describe which tasks resulted in pain, and share tips for injury prevention.

Results Themes identified through content analysis of transcripts include: a) Care giving relationship-The approach that HCWs take toward their consumers impacts the consumers' behaviours and may reduce injury risks, b) Exchanging information-HCWs want to learn about consumer conditions and assistive devices/ practices to help mitigate pain and improve mobility and c) Problem solving about devices/practices-HCWs have a stake in introducing devices, educating and negotiating with consumers to impact their movement and care.

Conclusions In summary, CHWs have insights into their work and should be supported to 1) identify risky tasks, 2) to document barriers and solutions to implement them, 3) to share resources and 4) to identify training needs. The findings may be explored in labour-management forums in order to create policies and procedures to reduce ergonomic risks to HCWs and their consumers.

\section{THE EFFECTS OF ACUTE AND CHRONIC MENTAL STRESS ON CARDIAC FUNCTION}

Mark Wilson, Lorraine Conroy. University of Illinois at Chicago, Chicago, IL, USA

\subsection{6/oemed-2014-102362.236}

Objectives The main objective of this study is to investigate the effects of chronic and acute stress on blood pressure, heart rate variability and an indicator of cardiac contractility. The study also aims to describe the degree to which various demographic and lifestyle factors modify the observed effect.

Method Chronic stress was assessed with a validated questionnaire, the Perceived Stress Scale. Acute stress was the level of stress experienced from the performance of a simple mental challenge on a scale from 1-100. Heart rate variability is calculated from heart rate measurements recorded via a heart rate monitor. Cardiac contractility index is derived from the amplitude ratio of the first to second heart sounds, as recorded with an electronic stethoscope. Measurements for each of the parameters were taken before and after performance of the task. Pretask measurements were used as baseline data and related to reported chronic stress levels. Acute stress levels were compared with the difference between pre- and post-task cardiac measurements.

Results Average acute stress level was 17/100. A statistically significant increase in cardiac contractility index was found for acute stress, while no significant change in either HRV or heart rate was noted. Subjects with high chronic stress showed a significantly reduced cardiac contractility than those with low chronic stress, while no difference in HRV was found between groups. Conclusions Acute and chronic stress exerts differing inotropic effects on the heart, while no chronotropic effect was observed.

\section{WORKPLACE VIOLENCE AND DEPRESSIVE SYMPTOMATOLOGY AMONG POLICE OFFICER}

${ }^{1}$ Sang Gil Lee, ${ }^{2}$ Inah Kim, ${ }^{3}$ Daeho Kim. 'Occupational Safety and Health Research Institute, Korea Occupational Safety and Health Agency, Incheon, Republic of Korea; ${ }^{2}$ Department of Occupational Health, Graduate School of Public Health, Yonsei University, Seoul, Republic of Korea; ${ }^{3}$ Occupational Lung Diseases Institute, Korea Workers' Compensation and Welfare Service, Gyeonggi-Do, Republic of Korea

10.1136/oemed-2014-102362.237
Objectives The prevalence of depression in police officer is higher than in the general population. Police officers are often exposed to work place violence, long time work, shift work and other pcychosocial stressers. This study was conducted to evaluate the occupational factors associated with depressive symptomatology in police officer.

Method The study sample was 7476 police officers. A structured questionnaire was used to assess exposures to violence, shift working with jobs, health behaviours and sociodemographic factors. Subjects with depressive symptomatology was who experienced depressive symptom for continuously for more than 2 weeks within last 1year. And chronic work place violence exposed subjects was who experienced threat or complaint from work related people more than 4 times per week.

Results Prevalence of depressive symptom among subjects was $35.5 \%(\mathrm{n}=2622)$ and higher in subjects exposed to chronic violence. After adjusting covariates, the odds ratio of depression was 2.01 (95\% CI; 1.80-2.25) for chronic work place violence exposed subjects $(\mathrm{n}=2005,27.11 \%), 1.20$ (95\% CI; $1.02-$ 1.41) for shift working subjects $(n=6270,85.68 \%)$.

Conclusions Our findings support the association workplace violence exposure between depressive symptomatology. Further study with more detailed work place violence exposure classification and measurement is need to confirm the association workplace violence and depressive symptomatology.

\section{THE COMBINED EFFECTS OF HIGH EMOTIONAL DEMANDS AND LOW JOB CONTROL AT WORK ON SUICIDAL IDEATION IN KOREAN SALES AND SERVICE WORKERS}

1,2,3 Jin-Ha Yoon, ${ }^{3,4}$ Sei-Jin Chang. 'Institute for Occupational Health, Yonsei University College of Medicine, Seoul, Korea; ${ }^{2}$ Department of Preventive Medicine, Yonsei University College of Medicine, Seoul, Korea; ${ }^{3}$ Department of Preventive Medicine, Wonju College of Medicine, Yonsei University, Wonju, Korea; ${ }^{4}$ Institute Occupational and Environmental Medicine, Wonju College of Medicine, Yonsei University, Wonju, Korea

\subsection{6/oemed-2014-102362.238}

Objectives Suicide rates have increased worldwide, and suicide is the second leading cause of death below the age of 60 in Korea. Hence, loss of the working years due to suicide is very important issue in occupational health. We examined the effects of high emotional demand and low job control on suicidal ideation in sales and service workers.

Method A total of 1995 participants (824 men and 1171 women) were recruited in this study. Suicidal ideation, high emotional demand and low job control were estimated by self-report questionnaires from the 4th Survey. Odds ratios (OR) and 95\% confidence intervals $(95 \% \mathrm{CI}$ ) for suicidal ideation was calculated by gender using the logistic regression analysis.

Results People who experienced high emotional demands (OR: 2.07 in men, OR: 1.97 in women) or low job control (OR: 1.96 in men, OR: 1.33 in women) were more likely to experience suicidal ideation, after adjusting for age, household income, and employment characteristics (paid vs. self-employed workers). The combined effects of emotional demands and job control revealed that workers with high emotional demand and high job control (OR: 1.93 in men, OR: 1.60 in women) and high emotional demand and low job control (OR: 4.60, OR: 2.69 in women) had a higher risk for suicidal ideation, compared to workers with low emotional demand and high job control, after controlling for age, household income, 